\title{
Hypothetical Art and Art Education: The Educational Role of the Method of Hypothetical Artwork Modelling
}

JURIJ SELAN ${ }^{1}$

A hypothetical artwork is an artwork that exists only as a fictional creation of an art theorist. The explicatory powers of such hypothetical artworks are mainly used by an art theorist to reflect on an art theoretical issue under consideration. Such an artwork has an intriguing and paradoxical nature. On the one hand, it is only fictitious, but, on the other hand, it tries to function as a real token, persuading the reader to trust it as if it were a real artwork. Even though this kind of argumentation can be deceiving, as it presents a statement of real art on the basis of fiction, it has some important explicatory abilities that can be put to good use in the art educational process. In this case, the construction of the hypothetical artwork is handled as the construction of a theoretical model. The author calls such theoretical construction the method of hypothetical artwork modelling, and its result the hypothetical artwork model. Such a hypothetical artwork model can be usefully employed when one wishes to encourage the student to become fictionally involved in the process of creation of an artwork, thus giving him or her more personal experience of problems that accompany the process of creating a real artwork. When such hypothetical experience is gained, the student can more efficiently learn about the considered art issue. In the paper, the author demonstrates how the explicatory powers of the method of hypothetical artwork modelling can be put into educational practice regarding an issue taken from colour theory (i.e., the primary colours fallacy).

Keywords: Art education, Art theory, Colour theory, Hypothetical art, Models, Primary colours

1 Faculty of Education, University of Ljubljana, Kardeljeva ploščad 16, 1000 Ljubljana, Slovenia jbselan@yahoo.com 


\section{Introduction: The Curious World of Hypothetical Art}

A hypothetical artwork is an artwork that exists only as a fictional creation of an art theorist (Selan, 2008, 2010). Usually it is introduced into the text by the linguistic assertion of supposition; for example, by the use of syntagms: »let us suppose, imagine that, etc. "Since the hypothetical artwork has no life outside the hypothetical world of a particular art theory, its intention is not to fascinate but to explicate. Therefore, the explicatory powers of hypothetical art are used by an art theorist mainly to reflect on a particular art theoretical issue under consideration and to verify certain stated art theoretical claims. One such famous hypothetical artwork is, for instance, Picasso's Le Cravat, which was invented by art theorist Arthur C. Danto (1981, p. 40).

However, a hypothetical artwork has an intriguing and paradoxical nature, which can be demonstrated as follows.

For a thing to be an artwork, two criteria that we routinely take for granted must be met. The first is the criterion of reality, which postulates two kinds of reality. On the one hand, the reality of facture: we believe that an artwork must be manufactured as a sensual artefact. ${ }^{2}$ On the other hand, there is the reality of place: an artwork must also be accepted by some cultural context (i.e., artworld) that evaluates it as an artwork.

This first criterion further implies the second: an artwork's reality must be an outcome of two modes of experience that come in a certain natural order. An artwork must first be created by an artist. Only later can an interpretation follow, which places it in the artworld.

If something does not meet these criteria then it is not an artwork and can find no place in any kind of art history. So, which are the »things « that do not succumb to these criteria?

First, let me take as an example the ordinary stone. We obviously do not treat it as an artwork and no art histories would document it as such. Even though it has a facture, it is not properly created; and even though it has a natural place, it is not socially placed, for it lacks a proper interpretation. In brief, it is, as Arthur C. Danto (1981, pp. 1-33) would say, just a "mere real thing. « However, as $20^{\text {th }}$ century art showed us, such a mere thing can actually at some point be accepted as an artwork, but only if properly transformed into a readymade.

2 Of course, I am not suggesting that all art concerns the manufacturing of material objects (e.g., performance art, conceptual art, body art, digital art, etc.). However, I want to stress that all art has to be presented in some kind of medium. Therefore, I use terms »facture " and »artefact, « (Latin: »facere« = 'make, create'), to accentuate this creational aspect that all artworks share. 
But what about a »thing « such as a hypothetical artwork? Could it be documented by some art anthology, such as that of David Summers (2003), where he precisely states the two categories of the artwork's reality: the facture and the place? No, for it lacks something that we all generally expect a real artwork to have. If a stone mostly lacks a proper interpretation and is thus a »mere thing, " a hypothetical artwork is only a fiction having no life outside its theory. Thus, it obviously lacks a facture and a proper creation, consisting only of an interpretation, and can for that reason be called, paraphrasing Danto's own expression, a »mere no-thing."

When such a mere no-thing is used in the deliberation of a particular art issue and in the argumentation of a stated art thesis it inevitably faces a paradox. On the one hand, it is only fictitious, but, on the other hand, it tries to function as a real token, persuading the reader to trust it as if it were a real artwork. Despite being fictitious, a »mere no-thing « has an intention to be taken as real.

It is precisely in this paradoxical nature that both the explicatory abilities and the limits of hypothetical artworks originate.

When an art theorist wants to address a particular art theoretical issue by constructing a hypothetical artwork, he or she must do so in a process of argumentation that is sufficiently logical to make the reader trust the constructed hypothetical artwork with the same certitude as if it were a real artwork. Such a process has two levels: inductive and deductive. Firstly, a hypothetical artwork must be established as a piece of evidence such as a real artwork would normally be. In order to do this, the art theorist must convince the reader that the constructed hypothetical artwork corresponds to the criteria to which real artworks spontaneously correspond. Since it is the real facture and the real artistic process that a hypothetical artwork critically lacks, the art theorist must simulate a creative process that could lead to the suggested artwork and its facture in reality. When the reader is convinced in this sense the hypothetical artwork can be taken as a valid piece of evidence in the argumentation of art problems that exist in reality.

Only when this is achieved can the hypothetical artwork enter the second level, where it is involved in a process of so-called (inductive) generalisation. In this process, the art theorist tries to argue that the thesis proved by some hypothetical artworks is generally valid for all artworks in history.

However, the problem is that such argumentation can go wrong-just as any kind of argumentation based on fiction can. Logical fallacies can appear at any of the two levels. I will try to demonstrate this with an example.

When on the first level of argumentation a hypothetical artwork is established as a reliable piece of evidence, this can be achieved in several different 
ways. Arthur Danto's book The Transfiguration of the Commonplace is full of such ways. Let me just consider the following one (Danto, 1981, p. 31): »Imagine that we learned that the object before us, looks like a painting that would spontaneously move us if we believed it had been painted-say the Polish Rider of Rembrandt, in which an isolated mounted figure is shown midjourney to an uncertain destiny-was not painted at all but is the result of someone's having dumped lots of paint in a centrifuge, giving the contrivance of a spin, and having the result splat on canvas, 'just to see what would happen'.«

This hypothetical artwork can be called the Polish Rider Made in a Centrifuge. The Polish Rider actually exists as an artefact. However, Danto transforms it into the hypothetical artwork by making speculations about how it could be created. So, by suggesting that the Polish Rider could also be made in a centrifuge, Danto tries to prove his basic thesis, which states: the facture of Polish Rider has no role in defining its artwork status; therefore, its entire value is defined exclusively by the social context or so called artworld.

However, such hypothetical artwork simulation does not convince me. Why? If I consider the daring hypothetical process that according to Danto could lead to the facture of the Polish Rider I cannot trust it. Just think about the actual possibility that the facture of the Polish Rider would be made in a centrifuge! It just does not seem plausible. Of course, some artworks-like those by Jackson Pollock or some brownish spills by Morris Louis-could more likely be made in a centrifuge. However, that only tells me that one cannot put factures of all artworks to the same denominator. Subsequently, from the point of view of reality, I cannot consider the Polish Rider Made in a Centrifuge as a piece of evidence that could properly justify Danto's argument, since it is impossible for it to really be made that way.

However, there is another problem that a hypothetical artwork can face in such argumentation. This problem is linked to the second level of the logical inference by (inductive) generalisation. Here the Polish Rider Made in a Centrifuge faces another logical issue.

Let us ask ourselves why Danto chose to ascribe the "centrifugal facture» to one of Rembrandt's artworks and not one by Morris Louis, which would have been better suited.

Danto's main intention is to generalise his basic thesis to all artworks in history. This generalised thesis states: the status of each and every artwork does not originate from its facture, which is thus irrelevant, but from the interpretation that locates an artwork in the proper place, called the "artworld « (Danto, 1981, pp. 124-126). However, real artworks (e.g., Duchamp's Fountain and Warhol's Brillo Boxes) that could prove such a claim have a limited range 
of generalisation. Because they belong to a narrow group of artworks that we usually denominate as "post-Duchampian «, they cannot inductively generalise Danto's thesis to all art. ${ }^{3}$ If Danto had made the inductive generalisation based only on post-Duchampian artworks this would have led him to the fallacy of hasty generalisation, which is a corollary of making inductive generalisations based on a range of evidence that is too narrow (Gardiner, 2008, pp. 139-140).

Thus, if Danto had wanted to prove his thesis for all art he would have had to resolve the problem of "too hasty generalisation. « This is precisely the intention of the Polish Rider Made in a Centrifuge: it enables Danto to also verify his thesis for the art of Rembrandt's time and subsequently expand the historical span of evidence to facilitate the logically valid inductive generalisation regarding all artworks in history.

However, as it seems to me, this does not work either. By constructing such a hypothetical artwork Danto only apparently avoids »too hasty generalisation «, while in fact making »too hasty generalisation « implicitly. When he constructs the Polish Rider Made in a Centrifuge he is already presupposing what he is only trying to prove: the general validity of his thesis for all art. This, however, leads such argumentation to the fallacy of circular argument, which presumes the truth of what is to be proved (Gensler \& Gensler, 2002, p. 328). Danto already takes for granted that which he is only trying to generalise by the Polish Rider Made in a Centrifuge. Otherwise, he would not construct a hypothetical artwork-one that is so obviously implausible in reality — in the first place.

\section{Hypothetical Artworks as Valid Models: The Method of Hypothetical Artwork Modelling}

The logical issues that I came across when reflecting on the Polish Rider Made in a Centrifuge made me recognise the cardinal problem that the hypothetical artwork must resolve in order to be taken as a valid piece of evidence: it must somehow submit to the criteria of good scientific argumentation.

As we all know, it is common and legitimate in serious science to use »mere no-things « to argue about certain scientific problems. However, it is

3 An inductive judgment is imperfect because it is based on a finite number of empirical pieces of evidence. However, when a wide enough repertoire of evidence is available to us we usually transform inductions into deductions by making inductive generalisations. In this sense, we have no doubt that the sun will raise tomorrow, even though we cannot be completely certain, as David Hume (1986, p. 20) once famously argued. 
important that in scientific argumentation such theoretically constructed mere no-things fulfil the essential criteria needed to construct something that logicians call a valid argument (Gamut, 1991, pp. 1-4). To simplify, two conditions are needed for a good argument: first, its premises have to be verified and accepted as true; and second, these premises must be further developed into a conclusion without digressing into logical fallacies.

In science, a premise or piece of evidence is accepted as true when it meets the condition of empirical verification in the reality of »our world «. So, when some pure theoretical constructions (»mere no-things «) are suggested as true they must also succumb to such verification. When they do, they become what the philosophy of science considers as theoretical models. In modal logic, such models are also understood as possible objects in possible worlds (Chellas, 1980, pp. 34-38). If an object presupposed in some possible world is to be rightfully considered as possible in our world too it must be established in accordance with the natural order of our world. That is, presuppositions of a possible world where a hypothetical artwork is constructed as a virtual piece of evidence must correspond to experiences in our world. Only when they do so can the constructed hypothetical artwork also be convincingly applied to a particular art issue in our world. So, we accept hypothetical artworks as valid models, and thus as true pieces of evidence, only if we can verify them in the reality of our world; if we cannot do so we reject them as invalid models and thus as untrue pieces of evidence.

As one can easily discover, it is precisely the verifiability of a possible object in the reality of our world that further prevents argumentation from getting into logical fallacies, such as the fallacy of circular argument. When an argument is circular, it is because its premises cannot be proven outside the possible world of the theory within which they were constructed.

From a scientific point of view, the Polish Rider Made in a Centrifuge is then nothing but an invalid model, because one cannot acknowledge the possible world in which it could be made as being compatible with our world. Therefore, if we wish to make good use of the explicatory powers of hypothetical art we must use it according to the criteria of good or valid argumentation. When it is done so, I call such theoretical construction of a hypothetical artwork the method of hypothetical artwork modelling and its result the hypothetical artwork model.

In the continuation of the article I will try to exemplify how hypothetical artworks can be constructed as valid models and subsequently also be put to good use in art education. The issue with regard to which I will explore the educational usefulness of the method of hypothetical artwork modelling is taken from colour theory and can be called the painters' primary colours fallacy (Selan, 2010). 


\section{An Example from Colour Theory: The Case of the Painters' Primary Colours Fallacy}

Painters traditionally considered red, yellow and blue (RYB) as primary colours, until on the grounds of physical and physiological discoveries in $19^{\text {th }}$ century it was proven that subtractive primary colours are actually cyan, magenta and yellow (CMY). However, whereas scientific colour theories took these new discoveries into account, artistic colour theories, such as those of Johannes Itten (1961) and Joseph Albers (1963), did not, which is why these theories were much criticised. Alan Lee, for instance, emphasised that it is excusable for Goethe (1840) not to accurately define primary colours since there was no exact knowledge back then on this matter, but that it is inexcusable for Albers and Itten not to have done so (Arnheim \& Lee, 1982).

My concern here is whether this kind of criticism regarding artistic colour theories is legitimate. I believe that it is inspired by the division that many scientific colour theorists, such as Harald Kueppers, insist on following when teaching about colour. They insist on the strict separation of colour theory, as a universally true science based on physical and physiological laws, from the history of colour theory and its incorrect scientific solutions, despite their experiential and cultural values (Kueppers, 2008).

Even though making such a distinction is generally necessary for valid scientific research, it seems to me that if we cling to it too strictly when teaching about colour this could lead us to some intolerable anomalies.

For instance, when Alan Lee criticised Albers's Interaction of Colour based on its theoretical fallacies he also put Albers' work as an artist under question (Lee, 1981). This, however, feels to me like an anomaly in argumentation, for one can at the same time learn a lot from the ingenious colour structures in Albers' work and, as Dorothea Jameson (1983) prudently replied to Lee, be aware of his theoretical fallacies.

Jonathan C. Fish (1981), for example, also criticised artistic colour theories and their primary colours fallacy. However, when he recognised that Piet Mondrian used the wrong primaries in his artistic work, he, unlike Lee, did not disprove the value of his art on that basis. »Turner and Mondrian succeeded superbly in their use of colour, despite their incorrect assumption, « he wrote (Fish, 1981, p. 91).

It was precisely the stated dilemma regarding artistic colour theories and their treatment of primary colours that stimulated me to reflect on the matter of the painters' primary colours fallacy more thoroughly. To do so, I used the method of hypothetical artwork modelling, by which I constructed the next two hypothetical artwork models. 


\section{Hypothetical Mondrian, Composition with Cyan, Magenta and Yellow}

Below is the first hypothetical artwork.

Presuppose, for example, the style of Piet Mondrian. To do so, one must decipher the "anatomy« of his real work (Kirsch \& Kirsch, 1988). He preferred vertical and horizontal lines, primary colours plus black and white, and golden section divisions for defining composition.

Now let me suppose one of his paintings. Because the issue I am concerned with is whether the primaries Mondrian used are right or wrong, I should give the hypothetical painting the working title Composition with $x, y$, $z$, where $\mathrm{x}, \mathrm{y}, \mathrm{z}$ stand for primary colours that are not yet defined but under consideration.

In the process of modelling hypothetical Mondrian I am then at some point forced to make a decision as to which $\mathrm{x}, \mathrm{y}, \mathrm{z}$ I should choose in order to get the best artwork.

Presumably following Lee, if one were hypothetically in the place of Mondrian one should decide on purely theoretical grounds and choose CMY primary colours. In this way one would get Composition with Cyan, Magenta and Yellow, or, for example, in the case of his famous Broadway Boogie Woogie, Broadway Boogie Woogie in Cyan, Magenta and Yellow.

However, as we know, the real Mondrian made a theoretical fallacy and chose RYB primaries instead, thus getting the theoretically wrong Composition with Red, Yellow and Blue and the Broadway Boogie Woogie as it is.

I should then ask myself: »What could justify such a decision by Mondrian?«

Could it be the recognition that the result of choosing theoretically correct CMY primaries would feel »wrong, « having a similar effect as neon art, and that RYB primaries would feel better?

If so, what is it that feels wrong about Composition with Cyan, Magenta and Yellow? Is it perhaps the wrong "moral « value of the CMY colour chord, as Goethe would probably claim? Let me compare, for example, the primary blues of CMY and RYB chords. The correct subtractive primary blue is cyan; historical review, however, shows us that painters traditionally treated the wrong ultramarine and cobalt blue as primary. Cyan blue is theoretically perfect for mixing, but in itself feels kind of superficial, electric and shallow, and has little cultural value. On the other hand, ultramarine and cobalt blue are incorrect as subtractive primaries, but what probably mattered to Mondrian is that they have more experiential deepness and therefore more exceptional spiritual and cultural significance than cyan. So the need for the right »moral«value of the 
colour chord would obviously make Mondrian prefer a historical choice of RYB primaries to the theoretical choice of CMY primaries.

The art educational value of the first hypothetical artwork comes forward instantly. Namely, while modelling a hypothetical Mondrian, a student of art can experience and subsequently recognise that the "right « choice of painters' primaries should not be subordinated only to the theoretical exactness of colour mixing, but should be also determined in relation to the historical tradition of colour usage and its experientially developed symbolism.

\section{Hypothetical Rembrandt, Double Self-Portrait, Colour Aquatint, Oil on Canvas}

The first hypothetical artwork model helped me transfer the accentuation from colour as a purely abstract entity in science to colour as the paint of cultural value in art. This, however, led me to construct a second hypothetical artwork.

Imagine that Rembrandt had both a painting enterprise and a colour printing enterprise. Now, let me suppose that he decided to create a diptych, which he would call Double Self-Portrait, made up of two identical pictures in terms of colour, differentiated only in the process of their facture, the first being made as a colour print and the second as a handmade oil painting. Given the framework of technological development of that time, Rembrandt would certainly have to do printing in etchings, maybe even aquatints, which was then being developed in the Netherlands (MoMA, 2008).

Concerning the issue of the primary colours fallacy, the next questions arise regarding the two presupposed hypothetical Rembrandts: Which primary colours would Rembrandt have to choose to get the identical result in both pictures of the diptych in terms of colour? Would these colours have to be the same? If not, why not?

Both for printers and painters, the question of primary colours was always concerned with the dilemma as to which pigments are actual material equivalents of primary colours. However, despite the fact that the interest of printers and painters in this matter appeared to be the same, primary colours fulfilled significantly different functions for each group.

A printer's aim is the complete colour prevision of an image and its infinite colour reproducibility; therefore, the printer must resolve two crucial problems regarding primary colours. Firstly, the function of printers' primaries must follow the strict axiomatic logic of colour mixing: as few as possible basic colours must intermix in all possible colour perceptions in an absolutely predictable and repeatable way. Secondly, material equivalents of such exact 
primary colours must result in minimal spectral defects $;{ }^{4}$ if not, the actual mixing of material pigments will not match the abstract axiomatic logic of colour mixing.

The first prints produced by J. C. Le Blon around 1738 show us that the time the choice of pigments was based on RYB primaries. Pigments like Prussian blue and dark yellow lake were used, and for red a mixture of madder-lake, carmine and cinnabar. As it turned out, these pigments did not match the rigorousness of printers' primaries, for the printing result was rather poor (Gage, 1993 , p. 162, 169, 216, 221-226). Of course, we know today that pigment equivalents of CMY primaries are needed to fulfil the function of printers' primaries, and not of RYB primaries. Thus, in his time, Rembrandt would have had no chance of making a colour print of the desired quality because the knowledge of exact printers' primaries was not properly developed until $19^{\text {th }}$ century.

Therefore, the first educational lesson of the presupposed hypothetical Rembrandt is related to the issue of the function of printers' primary colours. While modelling the hypothetical Rembrandt, a student of art can learn that without exact primaries and corresponding pigments successful colour printing could not have been developed. Thus, for printers the discovery of the principles of colour mixing and of synthetic colour pigments in the $19^{\text {th }}$ century, which enabled the materialisation of exact primaries, were of the outmost importance.

However, what about the educational lesson concerning the function of painters' primary colours? Obviously, Rembrandt painted successfully enough with the chosen range of pigments. A general historical review of painters' palettes enables reflection on which primary colours and paints he actually used (Gage, 1993, pp. 177-190). Rembrandt's palette is a typical Dutch tonal palette of the time, with paints ranging from white, orange-red, yellow-ochre, cinnabar red, several browns to several blacks (Gage, 1993, p. 178, 184, colour plate no. 145). There is almost no sign of blues, apart from some dark Prussian blue.

Obviously, Rembrandt's range of painting pigments was also equivalent to RYB primaries; however, in this case these pigments, which were inadequate

4 Because none of the material pigments can ever perfectly match the abstract exactness of primary colours, spectral defects occur when mixing primary pigments in practice. This means that in practice colour mixing of primaries does not result in colour mixtures (i.e., secondary colours) of the same intensity and saturation as should occur in theory. In theory-as is evident from colour wheels where secondary mixtures, like green, violet and orange, lie on the same circumference as primary colours - the intensity and saturation of primary and secondary colours should be the same. 
for colour printing, did their job well. Why is this so? What makes the difference? The reason lies in differentiating between the goals of printers' and painters' primaries. In contrast to printers, the painter's goal is not the repeatable colour reproduction of an image, which must be multiplied infinitely, but a unique artwork that should not be repeated. Therefore, painters need not rely only on a limited and unchangeable range of primary colours, but can use a repertoire of all of those pigments that can help them achieve the desired effect. Because painters do not have to prevision the effect of a picture in terms of colour with the intention of reproducing it infinitely, they do need not to control the colour mixing process of pigments with axiomatic precision. Consequently, for a painter colour mixing is not a strictly formalised and absolutely controllable process, but instead an intuitive and unpredictable creative flow, where spectral defects - a printers' worst nightmare - are not deficiencies that have to be annulled but rather the desired source of a painting's unpredictability and uniqueness, which can give the painting the mystic charm of being born uncontrollably. Thus, painters do not need to rely on exact primaries but can get along with approximate primaries just fine.

Modelling hypothetical Rembrandt can thus make a student of art experience that while pigment equivalents of RYB primaries were useless for printing, since printing demands a limited and fixed range of pigments, they functioned just fine in painting, where one can rely on a changeable and variable range of pigments.

The educational relevance of the two constructed hypothetical artwork models can be summed up as follows. Because these two hypothetical artworks draw attention to the essential difference in printers' and painters' primary colours, they can make a student of art experience that when evaluating the correctness of artistic colour theories, such as the theories of Goethe, Itten, Kandinsky, Klee and Albers, one cannot follow a strict division into theory and history of theory, for if one does so one must reject as wrong artworks that are in themselves entirely correct. Because printers were, due to the economic requirement of reproducibility, always preoccupied with the axiomatic problem of deriving complete colour perception from as few paints as possible, for them the principles of colour mixing discovered by developing science were of the utmost importance. On the other hand, painters at the beginning of $2 \mathrm{O}^{\text {th }}$ century did not need to share such enthusiasm about the newly discovered CMY primaries; instead, they could legitimately hold on to RYB primaries, which had a special historical and experiential value for them. Whereas printers must axiomatically derive complete colour perception from as few paints as possible, since they have to achieve the same result in each colour print, painters can 
adjust their pallets to the uniqueness of the painting they are creating. Consequently, also in painters' colour theories, which are rooted in painting practice and tradition, primary colours need not be fully subordinated to the axiomatic formalism of colour mixing, but should instead be seen more as practical approximations of colour mixing as an intuitive and rather unpredictable creative process. Thus, painters' primary colours should not be understood as axioms, but more as symbols-a kind of "holy trinity«—with the emphasis on their intense cultural significance (e.g., primary colours as a symbol of the Holy Trinity in Christianity), material significance (e.g., the different economic values of colour pigments; the spectral particularities of different colour pigments, etc.) and experiential significance (the different $»$ moral« value of different colour pigments).

\section{Conclusion: The Educational Value of Hypothetical (Art) Experience}

By the use of the method of hypothetical artwork modelling with regard to the example of the painters' primary colours fallacy I have tried to demonstrate hypothetical art's explicatory abilities, which can also be put to good use in the process of art education. I see its crucial explicatory, and thus also educational, advantage in attracting the student of art to become fictionally involved in the process of hypothetical artwork creation, thus giving him or her more of an experiential understanding of the art theoretical problems that accompany the process of creation of a real artwork. The usage of hypothetical art in art education can consequently transform art theoretical learning into a kind of experiential learning, since it enables the student to develop hypothetical experience, which can activate personal involvement and lived experience. When such hypothetical experience is gained, a student can reflect on and learn about the considered art issue in a more reasonable and realistic manner. This, however, prevents student's knowledge from losing its way in the anomalies and theoretical chimaeras that usually arise due to a lack of concrete and personal experience of problem solving in reality. 


\section{References}

Albers, J. (1963). Interaction of Colour. New Haven and London: Yale University Press.

Arnheim, R., \& Lee, A. (1982). A Critical Account of Some of Joseph Albers' Concepts of Color.

Leonardo, 15(2), 175.

Chellas, B. F. (1980). Modal Logic: An Introduction. Cambridge: Cambridge University Press.

Danto, A. C. (1981). The Transfiguration of the Commonplace. A Philosophy of Art. Cambridge:

Harvard University Press.

Fish, J. C. (1981). Colour as Sensation in Visual Art and in Science. Leonardo, 14(2), 91.

Gage, J. (1993). Colour and Culture. London: Thames and Hudson.

Gamut, L. T. F. (1991). Logic, Language, and Meaning: Introduction to Logic. Chicago: University of

Chicago Press.

Gardiner, J. H. (2008). The Making of Arguments. Charleston: Bibliobazaar.

Gensler, H. J., \& Gensler, H. J. (2002). Introduction to Logic. London: Routledge.

Goethe, J. W. (1840). Theory of Colours. London: John Murray.

Hume, D. (1986). A Treatise on Human Nature. London: Penguin Books.

Itten, J. (1961). Kunst der Farbe. Ravensburg: Otto Maier Verlag.

Jameson, D. (1983). Some Misunderstandings about Color Perception, Color Mixture and Color

Measurement. Leonardo, 16(1), 41-42.

Kirsch, J. L., \& Kirsch, R. A. (1988). The Anatomy of Painting Style: Description with Computer

Rules. Leonardo, 21(4), 437-444.

Kueppers, H.. Kueppers' Theory of Color. Retrieved December 30 2008, from http://www.ipsi. fraunhofer.de/Kueppersfarbe/en/theorie5o.html.

Lee, A. (1981). A Critical Account of Some of Josef Albers' Concepts of Color. Leonardo, 14(2), 99-105. MoMA. Retrieved December 30 2008, from http://www.moma.org/collection/details.php?theme_ id=10452\&section_id=Too3497.

Selan, J. (2008). Who's Afraid of Picasso's Tie, or Do you See the Catch in Hypothetical Art?

Leonardo, 41(5), 536-537.

Selan, J. (2010). Exploring the Method of Hypothetical Artwork Modelling: The Case of the Primary

Colours Fallacy. Leonardo, 43(3), 314-315.

Summers, D. (2003). Real Spaces: World Art History and the Rise of Western Modernism. London: Phaidon Press. 


\section{Biographical note}

Juris Selan, born 1982, graduated from the Academy of Fine Arts and Design in Ljubljana in 2005. Between 2006 and 2010 he was employed as a research assistant at the Academy of Fine Arts and Design where he obtained his PhD in Art Theory in 2011. Since 2010 he has been employed as an assistant at the Department of Art Pedagogy of the Faculty of Education in Ljubljana. 